\section{Sinistral nematode population}

SIR - Several animal taxa display a consistent left-right asymmetry of the body plan. In nematodes, dextrality predominates ${ }^{1}$. However, we have now found a nematode species that has sinistral populations.

In nematodes of the order Rhabditida, the anterior gonadal arm generally lies on the right of the intestine. Together with other asymmetries at the cellular level found in other nematode orders ${ }^{1,2}$, this defines the overall body handedness. After looking at many wild isolates of free-living soil nematodes, we found one population (strain PS1158) with reversed handedness. We identified it as Acrobeloides bodenheimeri Steiner, $1936^{3}$, Family Cephalobidae, Order Rhabditida. Type specimens for this species and for two independent isolates are also sinistral (see table). Other fixed and cultured isolates, previously identified by morphological criteria as $A$. bodenheimeri $^{4}$, are dextral (for example, strain PS2052). PS2052 crosses with neither PS1158 nor PS2160 (which themselves cross). PS1158/PS2160 and PS2052 thus form two biological species that are mirror images of one another at the cellular level. Because type specimens for $A$. bodenheimeri are sinistral, we conclude that this species name applies to PS1158/PS2160. (We will report elsewhere on the identity of the species represented by PS2052.)

Reverse handedness does not, as in some snails ${ }^{5}$, prevent mating per se: mating actually occurred in crosses between PS1158 and PS2052, as revealed by the copulatory plug left by the male over the female's vulva. Also, crosses between sinistral males and rare dextral females from PS1158 yielded progeny, and the converse was also true.

Handedness is apparent from the early cleavages of the nematode embryo, in the second division of the $\mathrm{AB}$ blastomere ${ }^{6}$. The axis of this division can be experimentally inverted in Caenorhabditis elegans, causing handedness reversal in the adults ${ }^{7}$. The respective fates of left and right blastomeres are set one cell cycle later by asymmetrical cell interactions ${ }^{8}$. In Acrobeloides sp. PS2052, as in other dextral nematodes, the left granddaughters of the $\mathrm{AB}$ blastomere are positioned anteriorly to their sisters $(8 / 8$ embryos). The converse is true in $A$. bodenheimeri PS1158 $(8 / 8)$ (see figure; in contrast to C. elegans, in these species the division of $\mathrm{AB}$ is delayed until P1 has divided three times). The asymmetry extends to the next cell cycle in the pattern of contacts to the blastomere MS. This is strikingly similar to the situation in snails, where the cleavage pattern reflects bodyplan handedness, and is only rarely reversed ${ }^{9,10}$.

We believe the present observation to be only the second example of handedness reversal of the whole body plan in an animal phylum. Reversal must be constrained, either because the transition is mechanistically difficult, or because of negative selection on reversed organisms $^{11}$. Our finding shows that the transition can nevertheless occur and leads to a complete reversal of the population, not only to an equal distribution of handedness.

Developmentally, cleavage handedness could be defined by some chiral cytoskeletal structure ${ }^{12-14}$. The evolutionary reversal of the cleavage pattern could act at this level, or in the inversion of some component of another embryo axis. We hope that this can be investigated in these

\section{SINISTRAL AND DEXTRAL NEMATODES}

\begin{tabular}{|c|c|c|c|c|}
\hline Designation & Status & Origin & Sinistral* & Dextral* \\
\hline PS1158 & In culture & California & 186 & 14 \\
\hline PS2160 & In culture & California & 198 & 2 \\
\hline A. bodenheimeri & $\begin{array}{l}\text { Type specimens } \\
\text { Steiner, } 1936\end{array}$ & Israel & 15 & 1 \\
\hline A. bodenheimeri & Fixed specimens & Libya & 10 & 0 \\
\hline PS2052 & In culture & Senegal & 2 & 198 \\
\hline 'A. bodenheimeri' & Fixed specimens & Malawi & 0 & 12 \\
\hline
\end{tabular}

We determined handedness of fixed specimens by the position of the gonad relative to the intestine (these species have a single gonadal arm extending anterior to the vulva). For living populations, handedness was determined on juveniles by the concordance of at least two criteria from ref. 7 . Details on the left-right asymmetries at the cellular level and on strain isolations are available on request. Gonad position in the type specimens was not explicitly mentioned by Steiner, but does appear in his original drawings ${ }^{3}$. Every female of PS1158, irrespective of its phenotype (or of that of the father), produces some dextral progeny, showing that handedness is not imprinted on the embryo by the phenotypic handedness of the mother. The changes in gonad position noted by Lorenzen ${ }^{15}$ outside the order Rhabditida could be indicative of changes in handedness of the whole-body plan but may well have evolved independently of handedness.

* Number of animals. closely related nematode species with opposite handedness.

Marie-Anne Félix

Paul W. Sternberg

California Institute of Technology,

Biology 156-29, Pasadena,

California 91125, USA

Paul De Ley

Instituut voor Dierkunde,

Ledeganckstraat 35,

B-9000 Gent, Belgium
. zur Strassen, O Verh dt zool Ges, 77-81 (1951).
2. Sulston, J. \& Horvitz, H. R. Devl Biol 56, 110-156 (1977).
3. Steiner, G. Proc. Helminth. Soc., Wash. 2, 74-80 (1936).
4. Siddiqi, M. R., De Ley, P. \& Khan, H. A. Afro-Asian J. Nematol. 2, 5-16 (1992).
5. Lipton, C. S. \& Murray, J. Malacologia 19, 129-146 (1979).
6. zur Strassen, O. Zoologica 107, 1-142 (1959).
7. Wood, W. B. Nature 349, 536-538 (1991).
8. Hutter, H. \& Schnabel, R. Development 120, 2051-2064 (1994)
Crampton, H. E. Ann. N. Y. Acad. Sci. 8, 167-169 (1894).
10. Vermeij, G. J. Nature 254, 419-420 (1975).
11. Gould, S. J. \& Young, N. D. Evolution 39, 1364-1379 (1985)
12. Guerrier, P. J. Embryol. exp. Morph. 23, 611-637 (1970). 13. Meshcheryakov, V. N. \& Beloussov, L. V. Wilhelm Roux's Arch. 177, 193-203 (1975).
14. Freeman, G. in Time, Space and Pattern in Embryonic Development 171-196 (Liss, New York, 1983). 15. Lorenzen, S. Veröff. Inst. Meeresforsch. Bremerh. Suppl. 7 (1981)

\section{Correction}

IN the Scientific Correspondence "Turing patterns in fish skin?" by Thomas Höfer and Philip K. Maini (volume 380, page 678 ), the lettering of two parts of the figure was incorrect: part $b$ should have been labelled as part $d$, and part $d$ as part $b$. Parts $a$ and $c$ are labelled correctly. 